\title{
Sex with aliens: out of space or out of mind?
}

Frances Kemperer reviews BBC2's Horizon programme of 3 December 1994

Extraterrestrial kidnapping is in vogue. Adozen books are available that detail accounts by alleged victims. A telephone survey of almost 6000 Americans revealed that many reported 'abduction by an alien'. BBC's Horizon programme sent Dr Susan Blackmore, psychologist and TV-troubleshooter for the paranormal, to investigate.

Accompanied by the ethereal sounds of a didgeridoo, North American 'abductees' described how small grey beings, usually with large heads and large black eyes, take their victims to their brightly-lit craft. There they investigate and perform experiments (often hybrid breeding experiments) on the bodies of their captive humans, before returning them to where they were before. As a frequent abductee described it: "if Im sitting reading a book when they come, Im put back reading a book". Importantly, the aliens usually try, with some success, to erase the victim's memory of the experience: technology indeed. Many of the 'abductions' were during or close to sleep.

'Abductees' may have complete recall of their terrifying experience. More commonly they remember only fragments, but under hypnosis recall details, or even discover memories of abduction, where previously they were aware only of an unexplained blackout. It is well recognised that hypnosis can facilitate the construction of false memories. But with or without hypnosis the stories of most subjects were strikingly similar-including among those who denied ever having heard another's account.

One hypnotist had seen over 400 clients for the recovery or development of abduction memories. He was convinced that a non-human intelligence was investigating us, but uncertain as to their purpose, since they appeared to be neither bodysnatchers nor saviours of the planet. A psychologist reported that interviews with abductees revealed these subjects to be essentially normal, albeit with a high propensity to fantasy and imagery. There was no evidence that any of these subjects was psychotic. One psychiatrist considered whether these experiences might be memories of trauma, perhaps sexual abuse, or dreams. But, as he pointed out, memories of abuse would replicate a particular abuse story, even if vague and general, and dreams are always highly individual. He concluded that it was more likely that the reported abductions were real. Dr Blackmore speculated whether the descriptions were a hoax (unlikely) or the interpretations of an experience produced by the brain. The non-sexual encounters were perhaps a sci-fi interpretation of the common experience of sleep paralysis.

The trump card was given to a neurobiologist. He described how mild temporal lobe 'misfirings', which are common, may produce a wide range of unusual sensations including leaving the body. floating and flying, a sense of presence, or sexual arousal. Using technology worthy of the alleged alien investigators he then exposed $\mathrm{Dr}$ Blackmore's temporal and parietal lobes to computer generated electro-magnetic fields until she felt an unseen and frightening force pulling at her leg.

But this programme was more than a detective investigation of aliens. This was a story of how people strive to make sense of the abnormal and how their interpretations are shaped by context and belief. In previous centuries some of the experiences reported by abductees would have been interpreted as activities of the devil, as visits of the succubus/incubus. Perhaps others would have been interpreted as angels: modern accounts of visitations by angels are as consistent and 'real' as descriptions of these more sci-fi life forms. The prevailing culture influences both the sufferer's, and the observer's, interpretation of the abnormal. What would you think if a patient on your ward described visitations by aliens? And what might others think if you reported being visited yourself?

Frances Klemperer, Psychiatrist, Community Help Service, rue St Georges 102, Box 20, 1050 Brussels, Belgium 